\title{
The $\mathrm{R} 482 \mathrm{Q}$ lamin $\mathrm{A} / \mathrm{C}$ mutation that causes
} lipodystrophy does not prevent nuclear targeting of lamin $A$ in adipocytes or its interaction with emerin

\author{
Ian Holt ${ }^{1}$, Lisa Clements ${ }^{1}$, Sushila Manilal ${ }^{1}$, Susan C Brown ${ }^{2}$ and Glenn E Morris ${ }^{*}, 1$ \\ ${ }^{1}$ MRIC Biochemistry Group, North East Wales Institute, Wrexham, LL11 2AW, UK; ${ }^{2}$ Neuromuscular Unit, Imperial \\ College School of Medicine, Hammersmith Hospital, London, W12 ONN, UK
}

\begin{abstract}
Most pathogenic missense mutations in the lamin A/C gene identified so far cause autosomal-dominant dilated cardiomyopathy and/or Emery-Dreifuss muscular dystrophy. A few specific mutations, however, cause a disease with remarkably different clinical features: FPLD, or familial partial lipodystrophy (Dunnigan-type), which mainly affects adipose tissue. We have prepared lamin A with a known FPLD mutation (R482Q) by in vitro mutagenesis. Nuclear targeting of lamin A in transfected COS cells, human skeletal muscle cells or mouse adipocyte cell cultures (pre- and post-differentiation) was not detectably affected by the mutation. Quantitative in vitro measurements of lamin A interaction with emerin using a biosensor also showed no effect of the mutation. The results show that the loss of function of R482 in lamin A/C in FPLD does not involve loss of ability to form a nuclear lamina or to interact with the nuclear membrane protein, emerin. European Journal of Human Genetics (2001) 9, 204-208.
\end{abstract}

Keywords: in vitro mutagenesis; nuclear lamina; BIAcore; Emery-Dreifuss muscular dystrophy; dilated cardiomyopathy; myoblast

\section{Introduction}

Mutations in the lamin $\mathrm{A} / \mathrm{C}$ gene are responsible for autosomal dominant forms of Emery-Dreifuss muscular dystrophy $^{1,2}$ (EDMD: OMIM No. 181350) and dilated cardiomyopathy $^{3,4}$ (OMIM No. 115200). Recently, specific missense mutations in the lamin $\mathrm{A} / \mathrm{C}$ gene have been shown to cause Dunnigan-type familial partial lipodystrophy ${ }^{5-7}$ (FPLD: OMIM No. 151660). Patients with this autosomal dominant disease experience adipocyte degeneration after puberty. Subcutaneous fat is lost from the limbs and trunk but excess fat is deposited in other regions such as the head and neck. Serious metabolic consequences may accompany FPLD, notably insulin resistance and diabetes mellitus.

The A-type lamins, A and C, are produced from alternatively spliced mRNA products of the gene on chromosome 1q21.3, lamin C being essentially a shorter form of lamin A. ${ }^{8}$

*Correspondence: GE Morris, MRIC Biochemistry Group, North East Wales Institute, Wrexham, LL11 2AW, UK. Tel: +44 1978 293214; Fax: +44 1978 290008; E-mail: morrisge@newi.ac.uk

Received 21 August 2000; revised 6 November 2000; accepted 15 November 2000
They are components of the nuclear lamina and are localised mainly at the nuclear rim where they interact with nuclear membrane proteins, such as emerin and LAP1, and with chromatin. ${ }^{9,10}$ A-type lamins are also found inside the nucleus $^{11,12}$ associated or co-localised with emerin ${ }^{13}$ and LAP $2 \alpha^{14}$ and their expression is developmentally regulated. ${ }^{15}$ Most reported lamin A/C mutations in FPLD affect codon 482 in exon $8(\mathrm{R} 482 \mathrm{Q}, \mathrm{R} 482 \mathrm{~W} \text { and } \mathrm{R} 482 \mathrm{~L})^{5-7}$ or nearby residues $\mathrm{K}_{486 \mathrm{~N}}{ }^{6}$ and $\mathrm{G}_{465 \mathrm{D}^{7}}$ in the globular tail domain. This concentration of missense mutations causing FPLD suggests that the affected region of lamin $\mathrm{A} / \mathrm{C}$ may have some specific function in adipose tissue, possibly involving interaction with an adipocyte-specific nuclear protein, for example. It has not been established whether the mutant lamin $\mathrm{A} / \mathrm{C}$ is produced in normal amounts and incorporated normally into the nuclear lamina. This would be difficult to study using FPLD patient biopsy material because, as in all autosomal dominant disorders, the unaffected allele will produce normal lamin $\mathrm{A} / \mathrm{C}$ and this may obscure any changes in lamin $\mathrm{A} / \mathrm{C}$ caused by the mutation on the affected allele.

Emerin is the affected protein in the X-linked form of EDMD. ${ }^{16}$ It is a type II integral membrane protein which is 
anchored to the inner nuclear membrane by its hydrophobic C-terminal tail and projects into the nucleoplasm. ${ }^{17,18}$ Emerin and lamin A have been shown to co-localise in cells ${ }^{13}$ and tissues ${ }^{19}$ and to interact directly with each other. ${ }^{20}$ In fibroblasts from lamin A/C knockout mice, there was a partial loss of emerin from the nuclear envelope and a more general distribution in the endoplasmic reticulum, showing that correct emerin localisation is dependent upon A-type lamin expression. $^{21}$

We now present a study of the effects of a typical FPLD mutation (R482Q) on the subcellular distribution of lamin $A$ and its interaction with emerin. Wild-type and mutant lamin A, created by in vitro mutagenesis, were transfected into mammalian cells and tracked specifically using antibodies against an epitope tag. Bearing in mind the tissue-specific effects of the mutation, transfections were performed on pre-differentiation and post-differentiation mouse adipocytes, as well as with COS-7 cells and differentiating human myoblast cultures. As a first step in the study of effects of the mutation on the binding of lamin A to other nuclear proteins, interaction with emerin was studied by biomolecular interaction analysis on the BIAcore biosensor.

\section{Materials and methods}

Construction of eukaryotic expression plasmid and site-specific mutagenesis

Full-length human Lamin A cDNA in pMW172,22 a generous gift from Rob Moir, Helen Kent and Murray Stewart (MRC Laboratory of Molecular Biology, Cambridge, UK), was subcloned into pcDNA4/HisMax/C (Invitrogen, Groningen, Netherlands). Site-specific mutagenesis to change G1445 to A was performed using the MORPH site-specific plasmid DNA mutagenesis kit (CP Laboratories, Saffron Walden, UK) as described by the manufacturer. The mutagenic oligonucleotide, 5' CCTTGCTGACTTACCAGTTCCCACCAAAGTTCACC, with the mismatch (A1445) shown in bold was 5'-phosphorylated and purified by polyacrylamide gel electrophoresis (Amersham Pharmacia Biotech, Little Chalfont, UK). Plasmids containing the mutation were identified by DNA sequencing (Sequenase v.2.0; Amersham) with a primer $64 \mathrm{nt}$ upstream of the mutation (5'-CGCAACAAGTCCAATGAGG) and confirmed by dye-based automated DNA sequencing (Cambridge Bioscience Ltd., Cambridge, UK).

\section{Eukaryotic cell culture and transfection}

COS-7, an African green monkey kidney fibroblast cell line, was cultured in DMEM (Dulbecco's Minimal Essential Medium: Gibco BRL, Life Technologies, Paisley, UK) with $10 \%$ decomplemented horse serum (Gibco), 2 mM L-glutamine and antibiotics. Cells were seeded at $5 \times 10^{5}$ cells per glass coverslip $\left(22 \mathrm{~mm}^{2}\right)$ and grown to $60-90 \%$ confluency. Medium was removed and the cells washed once with culture medium prior to transfection.
Cells obtained from explanted human foetal muscle were cultured in Skeletal Muscle Cell Growth Medium (PromoCell $\mathrm{GmbH}$, Heidelberg, Germany). Before transfection, the medium contained 10\% foetal bovine serum (FBS) and this was reduced to 5\% FBS to promote myotube formation after transfection. 3T3-L1 mouse adipose cell line ${ }^{23}$ (kindly provided by Dr BA Ashton, The Orthopaedic Hospital, Oswestry, UK) was cultured in DMEM supplemented with either $10 \%$ decomplemented FBS or $10 \%$ decomplemented rabbit serum. The rabbit serum was used to promote adipocyte differentiation. After two days of culture, 3T3-L1 cells were fixed in $10 \%$ formalin and stained with $0.3 \%$ Oil Red $\mathrm{O}$ to show the lipid content. ${ }^{24}$

Plasmids for transfection were grown in E. coli NovaBlue cells, purified using EndoFree Plasmid Maxi Kits (Qiagen Ltd, Crawley, UK) and quantitated by UV spectrophotometry. Transfection was performed using SuperFect reagent (Qiagen). COS-7 and foetal muscle cells were transfected on coverslips but 3T3-L1 cells were transfected in suspension and then allowed to attach to coverslips. Two micrograms of plasmid DNA and $10 \mu \mathrm{l}$ SuperFect reagent was used for each coverslip. After $3 \mathrm{~h}$, cells were washed once with culture medium and fresh medium was added. Culture was continued for a further two days, after which cells were fixed in 50:50 acetone-methanol for $5 \mathrm{~min}$ and stored at $-80^{\circ} \mathrm{C}$.

\section{Immunofluorescence}

For immunohistochemistry, coverslips were brought to room temperature and the cells washed four times with casein buffer $(0.1 \%$ casein in $154 \mathrm{~mm} \mathrm{NaCl}, 10 \mathrm{~mm}$ Tris, $\mathrm{pH}$ 7.6). Cells were incubated with $1 \mu \mathrm{g} / \mathrm{ml}$ monoclonal anti-Xpress antibody (Invitrogen, Groningen, Netherlands) for $1 \mathrm{~h}$ at $37^{\circ} \mathrm{C}$ and washed five times with casein buffer. They were then incubated in the dark with fluorescein-conjugated horse anti-mouse IgG (20 $\mu \mathrm{g} / \mathrm{ml}$ : Vector Labs., Burlingame, CA) for $1 \mathrm{~h}$ at $37^{\circ} \mathrm{C}$ and then washed four times with PBS and once with distilled water. Coverslips were mounted in Hydromount (BDH Merck) and examined with a Leica DMLB epifluorescence photomicroscope. Images were captured using an integrating camera and frame-grabber under comparable exposure conditions.

\section{Expression and extraction of recombinant proteins}

Wild-type and R482Q mutant lamin A recombinant proteins were produced by subcloning the cDNAs from pcDNA4/ HisMax into pET21a (which has a His-Tag at the C-terminal end). Full-length human emerin cDNA in pET17b produces a protein without a His-Tag and was kindly provided by Dr Don Love (University of Auckland, New Zealand). Plasmids were transformed into competent E. coli BL21(DE3) cells and induced with isopropyl- $\beta$-D-thiogalactopyranoside (IPTG) as previously described. ${ }^{19}$ The proteins were purified from inclusion bodies by sequential extraction with 2, 4 and $6 \mathrm{M}$ urea in PBS. 
Protein interaction by biomolecular interaction analysis (BIA)

The BIAcore biosensor detects changes in total mass at the surface of a sensor chip by measuring variations of the critical angle needed to produce total internal refraction. The critical angle is dependent on the refractive index of the medium that light has to pass through. An increase in mass bound to the chip increases the refractive index and this increases the critical angle. The change in critical angle is proportional to the amount of bound protein, and is expressed as resonance units (RU). The shift in RU is plotted against time and is displayed as a sensorgram. Rabbit anti-mouse IgG was covalently attached to a research grade CM5 sensor chip by amino coupling according to the manufacturer's instructions (BIAcore AB, Stevenage, Hertfordshire, UK). The BIAcore-X apparatus was used with an operational flow-rate of $20 \mu \mathrm{l}$ per minute and all injected volumes were $70 \mu \mathrm{l}$. Mouse monoclonal anti-His-Tag (Sigma) diluted 1/100 in BIAcore buffer (10 mм HEPES, pH 7.4, 150 mm NaCl, 3.4 mm EDTA, 0.005\% P20 surfactant) was captured onto the rabbit anti-mouse IgG chip and subsequent binding of lamin and emerin in BIAcore buffer was performed as described previously. ${ }^{20}$

\section{Results}

The first published FPLD mutation in lamin A/C was $\mathrm{R} 482 \mathrm{Q} .{ }^{5}$ To determine the effect of this mutation on nuclear targeting, full-length lamin A cDNA was sub-cloned into the mammalian expression vector pcDNA4/ HisMax and the g1445a mutation was introduced by a site-directed mutagenesis method ('MORPH') that does not involve PCR. Successful mutagenesis was confirmed by automated DNA sequencing.

Cells derived from explanted human foetal muscle were cultured, transfected with either wild-type or mutant lamin A and allowed to fuse and form multinucleated myotubes. The expression of transfected protein was almost entirely nuclear and no difference in distribution was observed between wildtype (Figure 1A) and mutant lamin A (Figure 1B). Many of the nuclei with transfected lamin A were inside differentiated myotubes (Figure 1C,D), though these may derive from myoblasts fusing after transfection. Transfection efficiencies were about $10 \%$ for both wild-type and mutant lamin A. Both wild-type and mutant lamin A was found almost exclusively at the nucleus in at least $90 \%$ of transfected cells. Similar results were obtained with the transfection of COS-7 cells (not shown). The expression was usually even and intense at the nuclear rim with some intra-nuclear expression. Intranuclear expression of lamins is commonly observed, even for endogenous lamins. ${ }^{12,13}$ In addition to the nuclear expression, some cytoplasmic, mesh-like expression was observed, but in less than $10 \%$ of transfected cells for both wild-type and mutant lamin A (not shown). Such ectopic cytoplasmic expression is not uncommon for over-expressed proteins, including lamins. $^{25}$
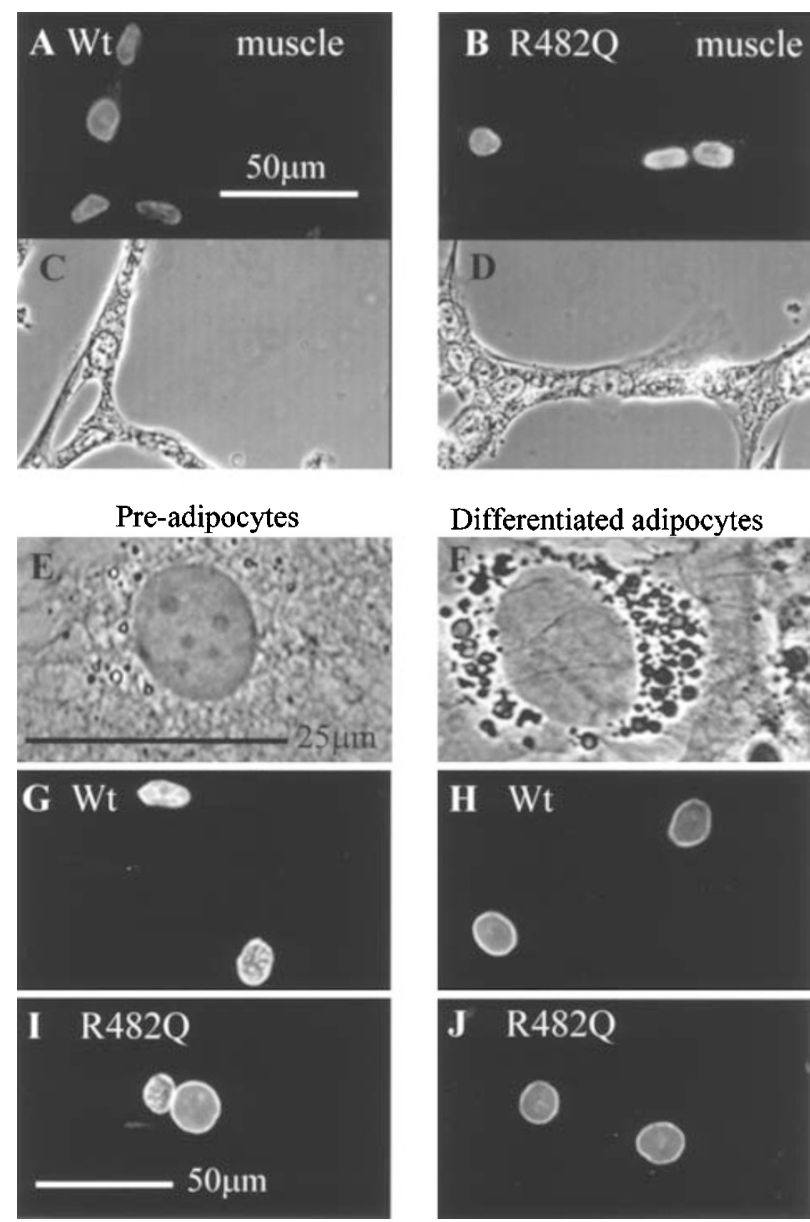

Figure 1 Localisation of transfected lamin A in human foetal muscle cells and in 3T3-L1 adipocytic cells is unaffected by the R482Q mutation. Transfected wild-type (A) and mutant (B) lamin $A$ in human foetal muscle cells were detected with antiXpress $m A$. Below the immunofluorescence images are the same fields under phase contrast (C,D). 3T3-L1 cells were cultured in either FBS $(\mathbf{E}, \mathbf{G}, \mathbf{l}$; pre-adipocytes) or rabbit serum $(\mathbf{F}, \mathbf{H}, \mathbf{J}$; differentiated adipocytes). (E,F) were stained with Oil Red $\mathrm{O}$ to show the increase in lipid vesicle content (black in phase contrast) after differentiation. Transfected wild-type $(\mathbf{G}, \mathbf{H})$ and mutant $(\mathbf{I}, \mathrm{J})$ lamin $\mathrm{A}$ are shown by immunofluorescence.

To study the effect of the mutation in a cell type of more relevance to FPLD, the 3T3-L1 mouse adipose cell line was studied at different stages of differentiation. The differentiation of 3T3-L1 cells was induced by rabbit serum. Free fatty acids in rabbit serum cause an increase in the proportion of Oil Red $O$ stained cells and an increase in mRNA of the adipocytic marker aP2. ${ }^{24}$ 3T3-L1 cells were cultured for two days in the presence of $10 \%$ FBS or $10 \%$ rabbit serum. Oil Red O staining showed low levels of lipid in cells cultured in FBS (Figure 1E) but a large number of lipid vesicles in cells cultured in rabbit serum (Figure 1F). The transfection efficiency of the 3T3-L1 cells was 2 to $5 \%$. This low efficiency 
compared with muscle cells and COS-7 cells could be a feature of either the cell type or the method of transfection. Muscle cells and COS-7 cells were transfected while they were attached to glass coverslips. However, this transfection procedure caused many of the 3T3-L1 cells to detach. Therefore, transfection was performed on 3T3-L1 cells while they were in suspension and they were subsequently allowed to adhere to the coverslips. As seen with the other cell types, the vast majority of 3T3-L1 cells transfected with either wildtype or mutant lamin A (>90\%) showed even and intense expression at the nuclear rim with some intra-nuclear expression. No difference in the distribution of either transfected wild-type or mutant lamin A was observed in those 3T3-L1 cells cultured in either FBS (Figure 1G,I) or rabbit serum (Figure $1 \mathrm{H}, \mathrm{J}$ ). Similar results were obtained when the cells were cultured in FBS prior to transfection and then allowed to differentiate in rabbit serum afterwards (results not shown).

The direct interaction of either wild-type or mutant lamin A with emerin was assessed using the BIAcore biosensor. A mouse $\mathrm{mAb}$ against His-Tag was captured on a rabbit anti(mouse Ig) chip and recombinant wild-type or R482Q mutant lamin A were captured via their C-terminal His-Tags. Lamin A input concentrations were adjusted so that equal amounts of wild-type and mutant lamins were captured. Equal volumes of the same preparation of recombinant emerin were then injected and Figure 2 shows that there was no significant difference in the amount of emerin binding to the two lamins. Control experiments showed that emerin did not bind significantly in the absence of lamin A and that other recombinant proteins did not bind non-specifically to lamin A. ${ }^{20}$ Table 1 shows two groups of four paired experiments of the type shown in Figure 2. In each case, there was no significant difference between the emerin to lamin A ratios using wild-type and mutant lamin A. In the first group of experiments, an emerin preparation stored in $6 \mathrm{M}$ urea was

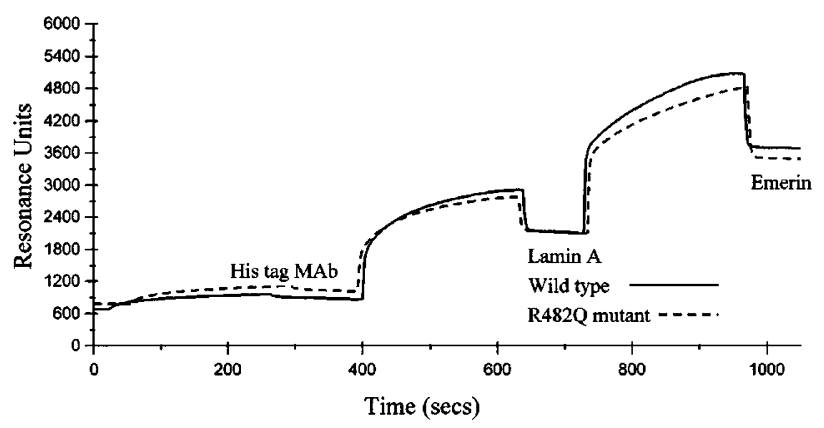

Figure 2 Binding of emerin to wild-type or R482Q mutant lamin A immobilised on a BIAcore sensor chip. An anti-His-Tag $m A b$ was used to capture either wild-type lamin A (solid line) or mutant lamin A (dashed line) on the biosensor chip. The high peaks as reactants cross the chip are due to refractive index changes. Reactant binding is measured by the baseline changes after excess reactant is washed away.
Table 1 Binding of emerin to wild-type and mutant lamin A

\begin{tabular}{llll}
\hline & $\begin{array}{l}\text { Emerin/ } \\
\text { wt lamin A }\end{array}$ & $\begin{array}{l}\text { Emerin/ } \\
\text { R482Q lamin A }\end{array}$ & $\mathrm{P}$ \\
\hline Experiment 1 & $\begin{array}{l}0.64 \pm 0.06 \\
(n=4)\end{array}$ & $\begin{array}{l}0.60 \pm 0.02 \\
(n=4)\end{array}$ & 0.60 \\
Experiment 2 & $\begin{array}{l}1.16 \pm 0.08 \\
(n=4)\end{array}$ & $\begin{array}{l}1.04 \pm 0.12 \\
(n=4)\end{array}$ & 0.41 \\
\hline
\end{tabular}

Results are expressed as the ratio of bound emerin/bound lamin $A$ ( \pm standard error on the mean of four BIAcore runs) determined from RU values as illustrated in Figure 2. Within experiments, the ratios of binding of the wild-type and the mutant lamin $A$ were not significantly different $(P>0.05$; two-tailed Student's $t$-tests). In experiment 1, recombinant emerin was stored in $6 \mathrm{M}$ urea and in experiment 2 in $4 \mathrm{M}$ urea. They were diluted about 100 -fold to $20 \mu \mathrm{g} / \mathrm{ml}$ with BIAcore buffer immediately before use. Lamin A preparations were stored in $6 \mathrm{M}$ urea and diluted about 50-fold to $10 \mu \mathrm{g} / \mathrm{ml}$ before use. (The difference in RU ratios between experiments 1 and 2 is explained in the text).

used and the emerin to lamin A ratio was lower than in the second group for which a preparation stored in $4 \mathrm{M}$ urea was used (Table 1). Urea was used to reduce aggregation and precipitation during storage and it is possible that the higher levels of emerin binding when the $4 \mathrm{~m}$ urea preparation was used reflect partial aggregation. The predicted ratio of emerin to lamin, based on $\mathrm{M}_{\mathrm{r}}$, for monomeric proteins of about 0.5 is close to the ratio obtained with the $6 \mathrm{M}$ urea emerin preparation (Table 1). Lamin A aggregation on the chip might reduce the ratio, but only if the emerin-binding sites were partially blocked in the aggregate.

\section{Discussion}

Rather than causing lipodystrophy, most reported lamin A/C mutations cause heart disease with characteristic conduction defects, either alone in dilated cardiomyopathy with conduction defects (DCM-CD) or with skeletal muscle problems in EDMD. It seemed possible, initially, that missense mutations within one specific region of the lamin $\mathrm{A} / \mathrm{C}$ rod domain cause DCM-CD while mutations outside this area cause EDMD. ${ }^{3}$ Further study with larger numbers of mutations, however, has shown that this distinction is not clear-cut. $^{2}$ Indeed, DCM-CD and EDMD can manifest in different members of the same family with the same lamin A/ $\mathrm{C}$ mutation, suggesting that genetic background can play an important role. ${ }^{2}$ The FPLD phenotype, however, appears to be so distinct from EDMD and DCM-CD that some adiposespecific function or interaction seems likely to be altered by the FPLD missense mutations.

The present study has not detected any differences between wild-type and mutant (R482Q) lamin A in nuclear targeting or in their interaction with emerin. These seemed reasonable hypotheses to test, since no data had been published on the distribution of mutant lamin A/C in FPLD patients and this is still to be established. It must be remembered that FPLD patients can be expected to produce 
normal lamin $\mathrm{A} / \mathrm{C}$ from the unaffected allele, as well as mutant lamin $\mathrm{A} / \mathrm{C}$ from the mutated allele, in this dominant disorder and it will be difficult to distinguish the two forms of the protein. The possibility that the protein product of the mutant allele is unstable and does not accumulate in adipocytes has not yet been ruled out in FPLD patients, though the transfection results presented here do not support this possibility. The lamin region required for emerin binding is not known, though there is no reason to suspect that the lamin tail domain is involved. LAP $2 \beta$, for example, binds to part of the helical rod domain (AA $78-258$ ) of lamin $B .^{26}$ It is also the rod regions of lamins that are involved in lamina assembly. ${ }^{10}$ The R482Q mutation may reduce lamin $\mathrm{A} / \mathrm{C}$ interaction with some protein other than emerin or lamins, possibly an adipocyte-specific nuclear protein, to produce its pathogenic effect. This interaction is unlikely to be required for correct nuclear localisation of lamin A in adipose cells, since the mutation does not affect localisation in either preor post-differentiation adipocytes.

\section{Acknowledgements}

This work was supported by grants from the EU Fifth Framework (contract QLRT-1999-00870) and the British Heart Foundation (PG97142). We are grateful to Louisa Brown (University of Dundee) for help with subcloning lamin A cDNA into pcDNA4 during a Wellcome Summer Studentship.

\section{References}

1 Bonne G, DiBarletta MR, Varnous S et al: Mutations in the gene encoding lamin A/C cause autosomal dominant Emery-Dreifuss muscular dystrophy. Nature Genet 1999; 21: 285-288.

2 Bonne G, Mercuri E, Muchir A et al: Clinical and molecular genetic spectrum of autosomal dominant Emery-Dreifuss muscular dystrophy due to mutations of the lamins A/C gene. Ann Neurol 2000; 48: 170-180.

3 Fatkin D, MacRae C, Sasaki Tet al: Missense mutations in the rod domain of the lamin A/C gene as causes of dilated cardiomyopathy and conduction-system disease. N Engl J Med 1999; 341: $1715-1724$

4 Brodsky GL, Muntoni F, Miocic S et al: Lamin A/C gene mutation associated with dilated cardiomyopathy with variable skeletal muscle involvement. Circulation 2000; 101: 473-476.

5 Cao H, Hegele RA: Nuclear lamin A/C R482Q mutation in Canadian kindreds with Dunnigan-type familial partial lipodystrophy. Hum Mol Genet 2000; 9: 109-112.

6 Shackleton S, Lloyd DJ, Jackson SNJ et al: LMNA, encoding lamin A/C, is mutated in partial lipodystrophy. Nature Genet 2000; 24: $153-156$.

7 Speckman RA, Garg A, Du F et al: Mutational and haplotype analyses of families with familial partial lipodystrophy (Dunnigan variety) reveal recurrent missense mutations in the globular C-terminal domain of lamin A/C. Am I Hum Genet 2000; 66: $1192-1198$
8 Lin F, Worman HJ: Structural organization of the human gene encoding nuclear lamin A and nuclear lamin C. J Biol Chem 1993; 268: 16321 - 16326.

9 Morris GE, Manilal S: Heart to heart: from nuclear proteins to Emery-Dreifuss muscular dystrophy. Hum Mol Genet 1999; 8: $1847-1851$.

10 Stuurman N, Heins S, Aebi U: Nuclear lamins: their structure, assembly, and interactions. J Struct Biol 1998; 122: 42-66.

11 Neri LM, Raymond Y, Giordano A et al: Spatial distribution of lamin A and B1 in the K562 cell nuclear matrix stabilized with metal ions. J Cell Biochem 1999; 75: 36-45.

12 Broers JL, Machiels BM, van Eys GJ et al: Dynamics of the nuclear lamina as monitored by GFP-tagged A-type lamins. J Cell Sci 1999; 112: 3463 - 3475 .

13 Manilal S, Nguyen thi Man, Morris GE: Colocalization of emerin and lamins in interphase nuclei and changes during mitosis. Biochem Biophys Res Commun 1998; 249: 643-647.

14 Dechat T, Vlcek S, Foisner R: Review: lamina-associated polypeptide 2 isoforms and related proteins in cell cycledependent nuclear structure dynamics. J Struct Biol 2000; 129: $335-345$.

15 Rober RA, Sauter H, Weber K, Osborn M: Cells of the cellular immune system and hemopoietic system of the mouse lack lamins A/C: distinction versus other somatic cells. J Cell Sci 1990; 95: 587-598.

16 Bione S, Maestrini E, Rivella S et al: Identification of a novel Xlinked gene responsible for Emery-Dreifuss muscular dystrophy. Nature Genet 1997; 8: 323-327.

17 Nagano A, Koga R, Ogawa $M$ et al: Emerin deficiency at the nuclear membrane in patients with Emery-Dreifuss muscular dystrophy. Nature Genet 1996; 12: 254-259.

18 Manilal S, Nguyen thi Man, Sewry CA, Morris GE: The EmeryDreifuss muscular dystrophy protein, emerin, is a nuclear membrane protein. Hum Mol Genet 1996; 5: 801-808.

19 Manilal S, Sewry CA, Pereboev A et al: Distribution of emerin and lamins in the heart and implications for Emery-Dreifuss muscular dystrophy. Hum Mol Genet 1999; 8: 353-359.

20 Clements L, Manilal S, Love DR, Morris GE: Direct interaction between emerin and lamin A. Biochem Biophys Res Commun 2000; 267: 709-714.

21 Sullivan T, Escalante-Alcalde D, Bhatt $\mathrm{H}$ et al: Loss of A-type lamin expression compromises nuclear envelope integrity leading to muscular dystrophy. J Cell Biol 1999; 147: 913-919.

22 Way M, Pope B, Cross RA, Kendrick-Jones J, Weeds AG: Expression of the N-terminal domain of dystrophin in E.coli and demonstration of binding to F-actin. FEBS Lett 1992; 301 : $243-245$

23 Green $\mathrm{H}$, Meuth M: An established pre-adipose cell line and its differentiation in culture. Cell 1974; 3: 127-133.

24 Diascro Jr DD, Vogel RL, Johnson TE et al: High fatty acid content in rabbit serum is responsible for the differentiation of osteoblasts into adipocyte-like cells. J Bone Miner Res 1998; 13: 96- 106.

25 Lourim D, Lin JJ-C: Expression of wild-type and nuclear localization-deficient human lamin A in chick myogenic cells. J Cell Sci 1992; 103: 863-874.

26 Furukawa K, Kondo T: Identification of the lamina-associatedpolypeptide-2-binding domain of B-type lamin. Eur J Biochem. 1998; 251: $729-733$. 